\title{
Revealing the therapeutic effects of aminolevulinate mediated femtosecond laser induced photo-chemotherapy in different cancer cells
}

\author{
Meltem Demirel Kars ${ }^{1, *}$, Gamze Yıldırım², Yasemin Gündoğdu ${ }^{3}$, Fatmanur Gönce ${ }^{2}$, Esra Ayan ${ }^{4}$, Hamdi \\ Şükür Kılıç
}

\begin{abstract}
Photodynamic therapy (PDT) is a photo chemotherapeutic strategy that is the application of photosensitizing agent and light on disease or tumor site. The aim of this study is to confirm the feasibility for femtosecond (fs) laser for aminolevulinate (ALA) mediated PDT on skin, breast and bladder cancer cells. Also the remarkable aspects of ALA mediated and laser induced PDT with respect to other literally known applications were investigated.

Metastatic melanoma cells SK-MEL30, mammary epithelial carcinoma cells MCF-7 and bladder cancer cells UMUC-3 were treated with ALA and then the cells were irradiated by fs laser at thirty wavelengths in between 230 and $800 \mathrm{~nm}$ for 30s and 60s. Anti-cancer effects of ALA phototherapy on different cancer cell lines were determined. Protoporphyrin IX (PpIX) accumulation was visualized by confocal microscopy. The effective PDT wavelengths were applied to evaluate the degree of apoptosis and necrosis in cells.

The viability tests demonstrated that wavelengths $400-440 \mathrm{~nm}$ and $600-630 \mathrm{~nm}$ were found to decrease the viability on three model cell lines. PDT at $630 \mathrm{~nm}$ exerted cell death by necrosis and apoptosis after $30 \mathrm{~s}$ and $60 \mathrm{~s}$ periods.

This paper confirms that ALA and femtosecond laser mediated PDT may be used together as therapeutic and diagnostic method to target breast, skin and urinary bladder cancer cells. The use of fs laser allows the flexibility for optimization of wavelength for photosensitizing agents.
\end{abstract}

Keywords: Aminolevulinate, apoptosis, MCF-7, Photodynamic therapy, SK-MEL-30, UMUC-3

${ }^{1}$ Department of Animal and Herbal Production, Meram Vocational School, Necmettin Erbakan University, 42090, Konya, Turkey

${ }^{2}$ Institute of Science, Nanotechnology and Advanced Materials Program, Selçuk University, 42075, Konya, Turkey.

${ }^{3}$ Department of Electric and Energy, Kadınhanı Faik İçil Vocational High School, Selçuk University, 42800, Konya, Turkey.

${ }^{4}$ Graduate School of Sciences and Engineering, Koç University, 34450, Istanbul, Turkey.

${ }^{5}$ Department of Physics, Faculty of Science, Selçuk University, 42075, Konya, Turkey.

${ }^{\star}$ Corresponding Author: Meltem D. Kars e-Mail: dmeltem@yahoo.com

Necmettin Erbakan University, AKEF Campus, B blok, No:410, Meram Yeniyol, Meram, Konya, Turkey.

DOI: $10.2478 /$ ebtj-2020-0024

(C) 2020 Authors. This work was licensed under the Creative Commons AttributionNonCommercial-NoDerivs 4.0 License.

\section{Introduction}

It has been proven more than 15 years ago that, rapid laser technology can be used to micro-structure materials by laser pulses very accurately (1). Femtosecond (fs) laser source develops very low thermal and physical deterioration to the neighboring material and tissue. This property leads the adaptation of fs laser technology to medicine. The usage of fs technology has been demonstrated in cornea shaping, photodynamic therapy, dental and ear surgery (2). The advantages of fs lasers in PDT include deep penetration of long laser wavelengths, the potential to reach the location of the tissue to be treated and decreased photo-bleaching of the photosensitizing agent (3). Photodynamic therapy has been proven to be an effective therapeutic way in dentistry for the treatment of periodontal pockets and osteo-necrosis in jaw (4). Photofrin (Porfimer Sodium) is a United States Food and Drug Administration (U.S. FDA) approved photosensitizing agent commonly used to treat esophageal cancer and non-small cell lung cancer by red laser induced PDT (5). So, PDT seems one of the novel therapeutic strategies deserving of work on for optimizing the parameters (6).

The therapy strategies for cancer patients with solid tumors have been developing through improvement of new generation drugs and therapeutic regimens (7). On the other hand, resistance acquired by tumor cells during chemotherapy develops demand to find other therapy protocols (8). One of the effective therapy protocols may be photody- 
namic therapy that the light source and photosensitizing agent stimulates the oxidative damage in tumor cells (9). PDT is a type of photo-chemotherapy which is based on the application of photosensitizer through systemic or topical ways and irradiation of the region by light sources. Photosensitizer and light interaction will develop reactive oxygen radicals in the cells. Porphyrin derivatives or porphyrin precursors may be used as photosensitizer and laser or incoherent light may be used as light source during PDT. Because malignant cells accumulate photosensitizer more than normal cells do, irradiation of the target region by light source will cause destruction of cancer cells preferentially due to highly reactive singlet oxygen accumulation and increased oxidative stress $(9,10)$. This feature makes PDT, a good targeted therapeutic strategy for cancer treatment. Photodynamic therapy provides the advantages like: tumor tissue specificity, prevention of resistance, multi PDT application on to the same tumor (10). ALA-PDT therapy is not developed very recently however the intensions of researchers for investigating the suitable photosensitizing material and its optimal concentration, light source and its effective intensity, and optimum wavelength, are going on. (11). Aminolevulinate (Aminolevulinic acid, 5-ALA) is metabolized to PpIX through heme pathway (12). PpIX activation induces the generation of singlet oxygen species in mitochondria and cell death is experienced $(13,14,15)$. 5-ALA has some potential advantages as photosensitizer as it is naturally occurring intermediate in the heme biosynthetic pathway, low amount of external ALA will be sufficient to induce PpIX. ALA also does not have intrinsic photosensitizer effect different from most of the photosensitizers and it has a more rapid photosensitizer clearance $(16,17)$. ALA-HCl has been approved by U.S. FDA as a drug to be used for brain tumor diagnosis on June 2017 (Gleolan, NX Development Corp.). Since 5-ALA is a naturally occurring metabolite not only in animal cells but also in some bacteria, it is produced by biotechnological methods in biorefinery concept (18). It is a potential valuable natural photosensitizing agent, so we focused on to establish the optimum wavelength for ALA-mediated fs-PDT to provide insight its usage as therapeutic tool and its dual function for cancer theranostics.

A Ti:Sapphire femtosecond (fs) pulsed facility was used to irradiate the tumor model cells. It provides the opportunity to work different wavelengths and to investigate the effects of altered wavelength on the efficacy of photodynamic therapy.

The aim of this study is to investigate the therapeutic effects of 5-ALA mediated and fs induced photodynamic therapy in breast cancer MCF-7, metastatic skin cancer SKMEL-30 and high grade bladder cancer UMUC-3 cells in time and wavelength dependent manner. The potential use of femtosecond laser at the most effective wavelength between Ultraviolet (UV, 230nm) - and Near Infrared (NIR, 800nm) in ALA-PDT in cancer therapy was evaluated. The use of fs laser provides the ease of working at varying wavelengths with single equipment for different photosensitizers having different absorbance features. The use of ALA- PDT by fs laser induction may lead a new theranostic tool for breast cancer, melanoma and high grade bladder cancer.

\section{Materials and methods}

\section{Cell-lines and conditions}

SK-MEL30 (cat.ATC151, DSMZ) melanoma cell line, UMUC3 (ATCC ${ }^{\oplus}$ CRL-1749 ${ }^{\text {Tx }}$ ) high grade bladder cancer cell line and MCF-7 mammary epithelial carcinoma cells were maintained as monolayer type cell culture in DMEM F12, MEM Earl's Medium and RPMI-1640 medium respectively (Figure 1). The cell culture medium $\mathrm{pH}$ was buffered to 7.0-7.4 both during culturing and during PDT. The culture medium was supplemented with $10 \%$ serum (FBS, v/v) and 1\% antibiotic solution (penicillin/streptomycin, v/v). When the adherent cells occupied 70\% of the cell culture vessel, they were transferred into new T25 flasks by mobilizing the cells with trypsin-EDTA. Three cells

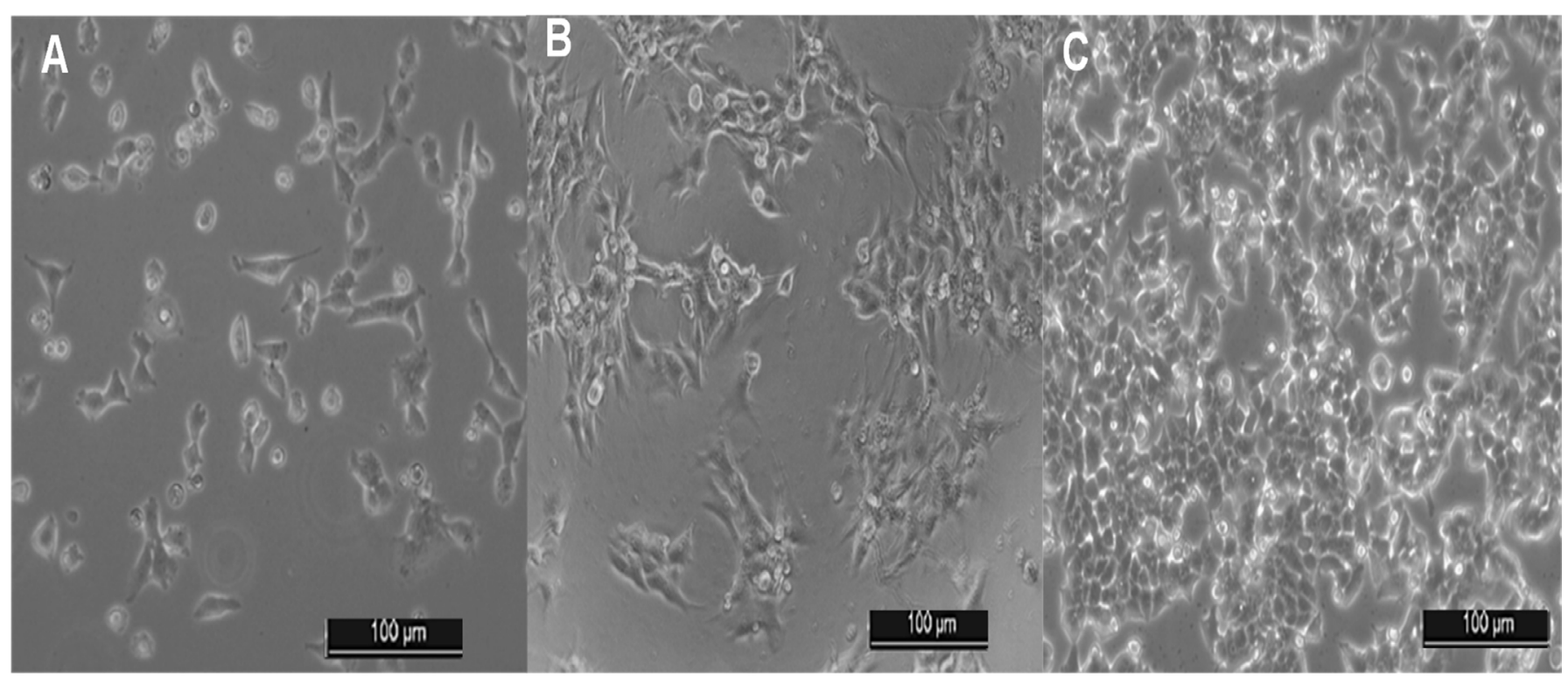

Figure 1. Cancer model cell lines: A) UMUC-3, B) SK-MEL30 C) MCF-7 (Invert, phase contrast light microscope) 
lines were studied at different times by different researchers (listed as co-authors) for this study.

Application of 5-ALA mediated photodynamic therapy to determine the optimum laser wavelength

A Ti:Sapphire fs pulsed laser system was used to irradiate the cells. It consists of three parts: an oscillator (Quantronix,Ti-Light, USA) laser that delivers laser pulses with $90 \mathrm{fs}$ pulse duration at $85 \mathrm{MHz}$ repetition rate at a wavelength in the near infrared region about 790-810 $\mathrm{nm}$ a; an amplifier (Quantronix, Integra-C, USA) system pumped by Ti-Light oscillator and used to amplify pulse energy, the output of which gives a $90 \mathrm{fs}$ laser pulse train with $1 \mathrm{kHz}$ repetition rate at $800 \mathrm{~nm}$ wavelength with an energy per pulse up to $3.5 \mathrm{~mJ}$ and; an OPA (Quantronix, Palitra, USA) system pumped by output of Integra-C delivering laser pulses at $90 \mathrm{fs}$ pulse duration over a wavelength range covering from UV to NIR in the spectrum. These facilities provided the opportunity to work wavelength dependence of light effect on the PDT. The beam area incident on the multi-well plates is about $0.5 \mathrm{~cm}^{2}$. The power of the light transmitted to inside of the 96-well plate was measured (45 $\mathrm{mW})$, eventually $0.09 \mathrm{~W} / \mathrm{cm}^{2}$ laser peak power density was applied. The spectral bandwidth of the laser is about $30 \mathrm{~nm}$. Hence the used wavelengths in this study were chosen taking into account this bandwidth. The applied laser fluence in this study was $9 \mathrm{x}$ $10-5 \mathrm{~J} / \mathrm{cm}^{2}$ with $1 \mathrm{kHz}$ repetition rate and $90 \mathrm{fs}$ pulse durations. These peak power density and also peak energy fluence values were applied for all used laser wavelengths on the cells both $30 \mathrm{~s}$ and 60s irradiations durations. During experimenting, laser energy was very stable and intensity of laser during the irradiation did not change for all wavelengths.

5-ALA was used as precursor for PpIX induction. SKMEL30, UMUC-3, MCF-7 cells were seeded to microplates (10,000 cells per each microwell) and incubated for attachment. 5-ALA ( $1 \mathrm{mM}$ final concentration) was added in to the growth medium ( $\mathrm{pH}$ 7.0-7.4) of microwells to be treated. To prevent the irradiation of neighbor wells, at least one row and one horizontal line wells were left empty from each side of the experiment wells. The $\mathrm{pH}$ of the medium is an important parameter that effects the PpIX production. The PpIX production requires pH 7.2 media. The cell culture medium contains sodium bicarbonate buffer system that works in the presence of $\mathrm{CO}_{2}$ in culture incubator (19). The micro plates were incubated for $4 \mathrm{~h}$ after treatment in dark to prevent toxicity from light, and then the well content was refreshed with medium that does not contain ALA (11). Cells were irradiated by thirty different wavelengths form UV to NIR (230 nm- $800 \mathrm{~nm}$ ) for 30 and 60 seconds. The irradiation was performed from the bottom of cell culture plates directing the laser light on to the each single well. Laser control wells attributed for each wavelength were not treated with 5-ALA, cell control wells were not applied 5-ALA and laser. The cells were incubated overnight. Each experiment sets were replicated three times.

Evaluation of the effects of photodynamic therapy with differ- ent fs laser wavelengths on the viability of skin, bladder and breast cancer cell lines

After the PDT treatment and incubation, XTT solution (XTT is a negatively charged, tetrazolium salt, Biological industries) was added to the wells and activated by activating solution, the cells were left for incubation for $4 \mathrm{~h}$ in $\mathrm{CO}_{2}$ incubator. Optical density of the plates were measured at $490 \mathrm{~nm}$ by a 96 -well plate reader (BioTek Instruments). The viability level of control cells was set to $100 \%$ and $\%$ viability of the ALA-PDT on cancer cells were determined from the mean of at least three results for each laser wavelength. The effective laser wavelengths, that inhibited SKMEL-30, UMUC-3 and MCF-7 cell viability after ALA-phototherapy, were determined. In this study, the PDT wavelengths was judged as "effective" when the viability of the cancer cells treated with ALA-phototherapy was found to be maximum $50-60 \%$. The cell viability tests generally aim to find the conditions that inhibit the fifty percent of the viability. So here we have chosen the conditions that the cell viability was $50-60 \%$ and lower to study the cell death mechanisms. Also control cell viabilities should be at high percentages (more than $60 \%$ ). We used the ALA-PDT conditions (laser wavelength) that resulted in maximum $50-60 \%$ cell viability to evaluate the cell death mechanisms in further experiments.

Evaluation of apoptosis and necrosis levels in melanoma, bladder and breast cancer cells after PDT

The most effective PDT wavelengths were determined by XTT assays as explained in previous sections. The validation of cell death mechanisms in those fs wavelengths were identified by analysis of apoptosis and necrosis by AnnexinV/ 7-AAD detection kits. Flow cytometry was used (FACS Aria III, BD, Canada) for fluorescent measurements. 10,000 cells from three different cell lines were seeded in microplates and incubated in $1 \mathrm{mM}$ 5-ALA containing medium (DMEM, EMEM or RPMI) one night. Output wavelength of fs laser system was set to the 630 and $420 \mathrm{~nm}$ for UMUC-3, $600 \mathrm{~nm}$ and $400 \mathrm{~nm}$ for SK-MEL-30 and $440 \mathrm{~nm}$ and $630 \mathrm{~nm}$ for MCF-7 cells and the cells were irradiated for 30 s and $60 \mathrm{~s}$ durations. After overnight dark incubation, attached and already detached SKMEL-30, UMUC-3 and MCF-7 cells after PDT were harvested from wells and the cells were treated with AnnexinVAPC (Biolegend) and 7-amino-actinomycin D (7-AAD, Biolegend) (11). The flow cytometer was adjusted for each cell line with unstained cells. Finally, fluorescence gates were analyzed according to the directions of staining kits. High 7-AAD staining quadrant (Q1, Q2) demonstrates death cells (due to apoptosis or necrosis), high Annexin-V (Q4) staining quadrant demonstrates apoptotic cells.

\section{Visualization of 5-ALA mediated fluorescent PpIX accumula-} tion

The intracellular fluorescent PpIX accumulation was observed by confocal microscope (CLSM, Nikon A1+). 200,000 cells model for different cancer tissues were seeded in glass bottomed cell view plates (Greiner) and the cells for incubated for 

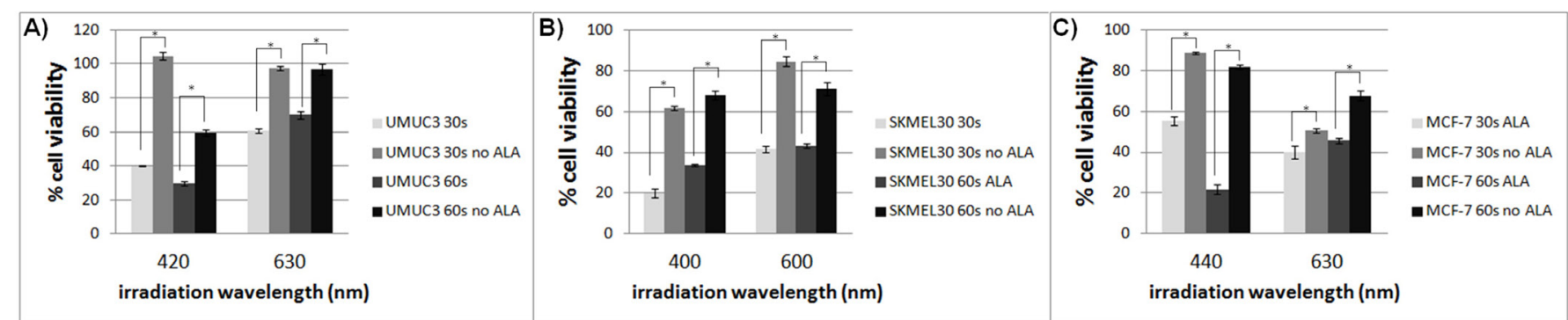

Figure 2. Cell viability profiles of the A) UMUC-3 cells, B) SK-MEL-30, C) MCF-7 cells at the selected PDT wavelength conditions. The significantly different groups were demonstrated by asterisk $(\mathrm{p}<0.05)$.

24 hours for attachment. Afterwards, the attached cells were treated with $4 \mathrm{mM}$ ALA in serum-free medium for 4 hours. The cell plates were washed with ice-cold PBS for three times, the fluorescence was detected in living cancer cells. The 5-ALA concentration and treatment duration was found non-toxic as previously tested (11). Light emission from PpIX was detected when the untreated wells were attributed as negative control. Control untreated cells and ALA treated cells were observed in white field by transmitted detector (TD) and then by confocal microscope with standard detector (SD) and TD. The confocal laser scanned images were analyzed by NIS Elements Viewer 4.0 (Nikon) then the images captured by TD and SD were merged to visualize the intracellular fluorescent accumulation (excitation: $405 \mathrm{~nm}$; emission: $614 \mathrm{~nm}$ ).

\section{Statistics}

The analyses regarding cell viability assessments were repeated at least three times and the significant variations between treatment groups were examined by applying Student's t-test $(\mathrm{p}<0.05)$.

\section{Results}

Effect of femtosecond laser mediated PDT on the viability of UMUC-3, SK-MEL-30 and MCF-7 cells

The PDT wavelengths were judged as effective when the viability of the cells treated with ALA-phototherapy was 50-60\% and lower. Additionally, the other key wavelength selection criterion was that the control group (without ALA) achieved at least $60 \%$ cell viability at the chosen wavelength. The viability tests on high grade urinary bladder, metastatic melanoma and breast cancer cells revealed that femtosecond laser irradiation with wavelengths lower than $400 \mathrm{~nm}$ resulted in low viability (lower than 50\%) in both control and ALA-PDT groups. Additionally, the treatment wavelengths between $440 \mathrm{~nm}-600 \mathrm{~nm}$ and $630-800 \mathrm{~nm}$ did not exert the effective cell death, which was more than $50 \%$ cell death. The only effective results (cell death more than $50 \%$ in PDT group and lower than $40 \%$ in control group) that passed the PDT criteria were obtained with $400-440 \mathrm{~nm}$ and $600-630 \mathrm{~nm}$ fs PDT in-vitro therapeutic protocol on model cell lines. Figure 2 demonstrates the cell viability profiles of the cells after PDT. According to the results, PDT decreased the viability of MCF-7 cells dramatically when compared to the other two more aggressive cancer cell models. Laser irradiation at UV wavelengths caused a decrease in viability of non-ALA cells. So, UV wavelengths seem to be not feasible for ALA-phototherapy protocol. The cell death in UV wavelengths may be due to the effect of UV light only. The viability of MCF-7 cells was decreased to $26 \%$ and 39\% after $440 \mathrm{~nm}$ fs laser PDT for $30 \mathrm{~s}$ and $60 \mathrm{~s}$ respectively. The cell viability was decreased to $40 \%$ and $45 \%$ when PDT at $630 \mathrm{~nm}$ wavelength was applied for $30 \mathrm{~s}$ and $60 \mathrm{~s}$ durations respectively. 420 $\mathrm{nm}$ PDT was the most effective therapy on high grade bladder cancer cell line which exerted $62 \%$ and $40 \%$ anti-proliferative effect after 30s and 60s irradiation respectively when compared to non-ALA laser treated control cells. 380-400 nm PDT was the most effective on metastatic skin cancer cell line which exerted $67-69 \%$ anti-proliferative effect after 30s and 60s irradiations when compared to no ALA laser treated control cells. Interestingly, 60s fs laser irradiated (non-ALA) UMUC-3 cell line viability was higher than non-treated control group. The inhibitory effects should also be investigated with further molecular analyses.

Flow cytometry analysis for the evaluation of apoptosis and necrosis levels in bladder, melanoma, and breast cancer cells after PDT

The flow cytometry analysis results exhibit the cell populations at different analysis quadrants (Figure 3-5). Q3 quadrant constitutes the living cells that were not stained by Annexin and 7-AAD. Q4 quadrant exhibits the cells that are apoptotic, phosphotidyl serine molecules of the apoptotic cell membranes were stained by fluorescent conjugated Annexin. Q1 and Q2 quadrants represent the cells that are in late apoptosis and in necrosis. In that case late apoptotic and necrotic DNA fragments were stained by 7-AAD. According to the flow cytometry results, there is considerable percentage of cell death in UMUC- 3 and MCF-7 populations after $420 \mathrm{~nm}$ and $440 \mathrm{~nm}$ PDT, respectively (Table 1). It seems that the $420 \mathrm{~nm}$ fs mediated, $1 \mathrm{mM} 5$-ALA PDT is the best therapeutic way that inhibits the viability of high grade UMUC-3 cells in-vitro. However the cell death of SKMEL-30 cells was not demonstrated well by flow cytometry apoptosis and necrosis analysis (Table 1). PDT 

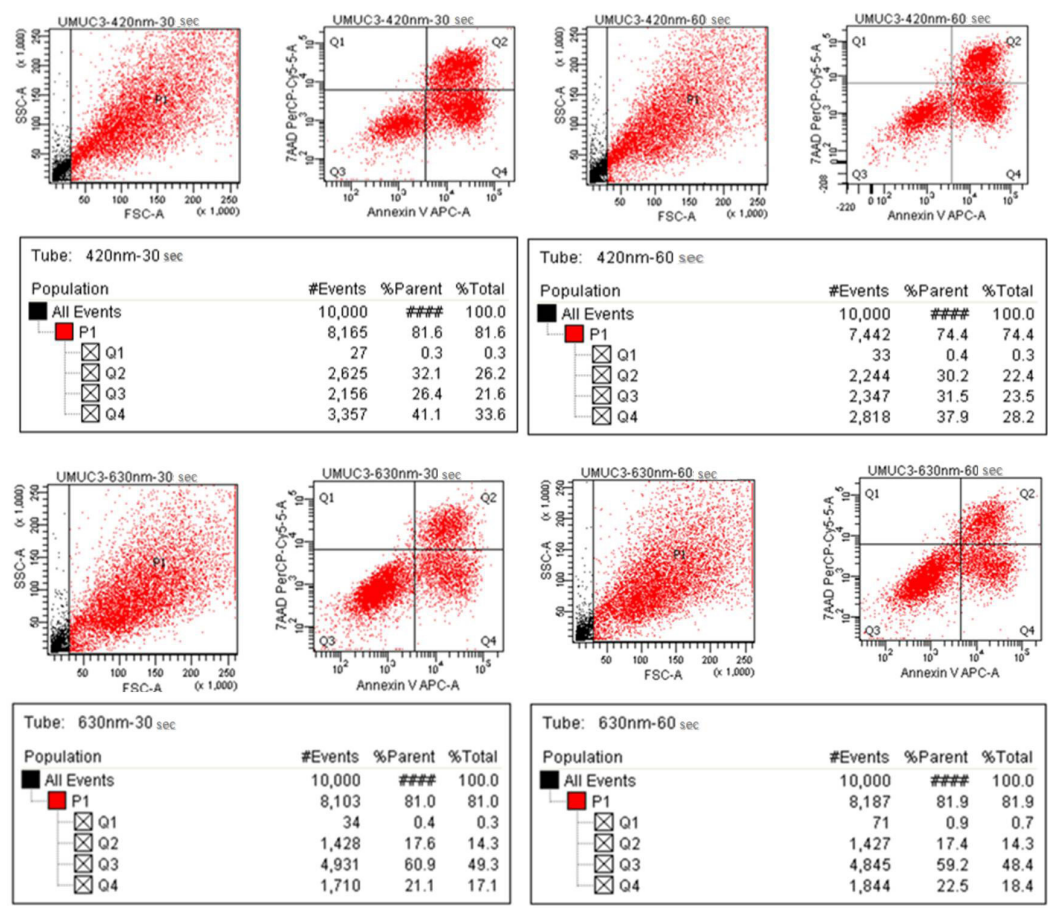

Figure 3. Annexin-V/7-AAD apoptosis/necrosis analysis of $30 \mathrm{~s}$ and $60 \mathrm{~s}$ PDT treated UMUC-3 cells.

at $440 \mathrm{~nm}$ for 30s and 60s durations induced cell death in about $70 \%$ of the MCF-7 population (Figure 5, Table 1).

\section{Confocal microscopy for detection of 5-ALA mediated fluores- cent PpIX accumulation}

The fluorescent PpIX accumulation after induction of PpIX with non-fluorescent 5-ALA was observed in three cancer model cells at the same conditions by confocal microscope (Figure 6). The cells that were not treated with 5-ALA were used as negative control. 5-ALA is not a fluorescent molecule itself so the observed fluorescence is due to the accumulation of fluorescent PpIX emission after induction of the cells with 5-ALA (20). Nontoxic concentrations of 5-ALA seems to be applicable for diagnosis of skin, bladder and breast cancer. Here the finding is both proof of the concept and it exhibits that three different cancer type cells may be diagnosed at the same conditions. Here it was shown that the non-toxic amount of 5-ALA (4mM) was enough to enhance the PpIX for visualization of three different cancer cells. Here this finding is important because it is known that PpIX was pumped out by cell membrane proteins in some circumstances (21). Especially the drug efflux pump ABCG2 is responsible for the chemotherapy resistance and PDT resistance in cancer cells. So, accumulation of PpIX is one of the most important parameters of successful PDT and also ALA mediated diagnosis in cancer.

\section{Discussion}

Efficacy of 5-ALA mediated photodynamic therapy with 30 different laser wavelengths in bladder, skin and breast cancer models was investigated in this study. The most effective PDT wavelength was determined by evaluating the cell viability analyses. Findings will provide new experimental biomedicinal results to the literature that were arisen from application of femto second laser at 30 different wavelengths on aminolevulinic acid treated bladder, skin and breast cancer cells. In our previous study we claimed that melanoma cells that were treated with fs induced ALA-phototherapy experienced apoptotic and necrotic cell death (11). In that work, the cells were excited by fs laser pulses $(10-15 \mathrm{sec})$ to target the light route, andalso it is known that fs-PDT does not cause any adverse effects to the nearby tissue (22). This treatment protocol may be very similar to the targeted therapy strategy. Three different model cell lines were used in this study and high grade bladder cancer cells and breast cancer cells were more sensitive to ALAPDT when compared to metastatic melanoma cells.

The influence of ALA-PDT on bladder cancer cells is considerable and promising in terms of the applicability of the treatment procedure to clinic. Bogoeva and his group applied Ruthenium (II) Porphyrin (RuP) mediated PDT with continuous blue light laser for 10 minutes on bladder cancer cells (23). They have found that cell survival was dramatically reduced to $14 \%$ after illumination of $20 \mu \mathrm{M} \mathrm{RuP}$ with $15.6 \mathrm{~J} / \mathrm{cm}^{2}$ blue light. They declared that the high percentage of cell population experienced necrosis. However the effect was not due to light source or photo-activation but the major effect was due to the toxicity of photosensitizer ruthenium porphyrin alone. In our study we found promising results with application of very short fs laser durations (30s, 60s). In our study generally, exposure durations of 30s and 60s did not exerted any significant differences between each other for the analyzed wavelengths of $400 \mathrm{~nm}$ and $600 \mathrm{~nm}$ bands. So we may propose that use the lowest exposure time as possible would be more advantageous. This would pro- 

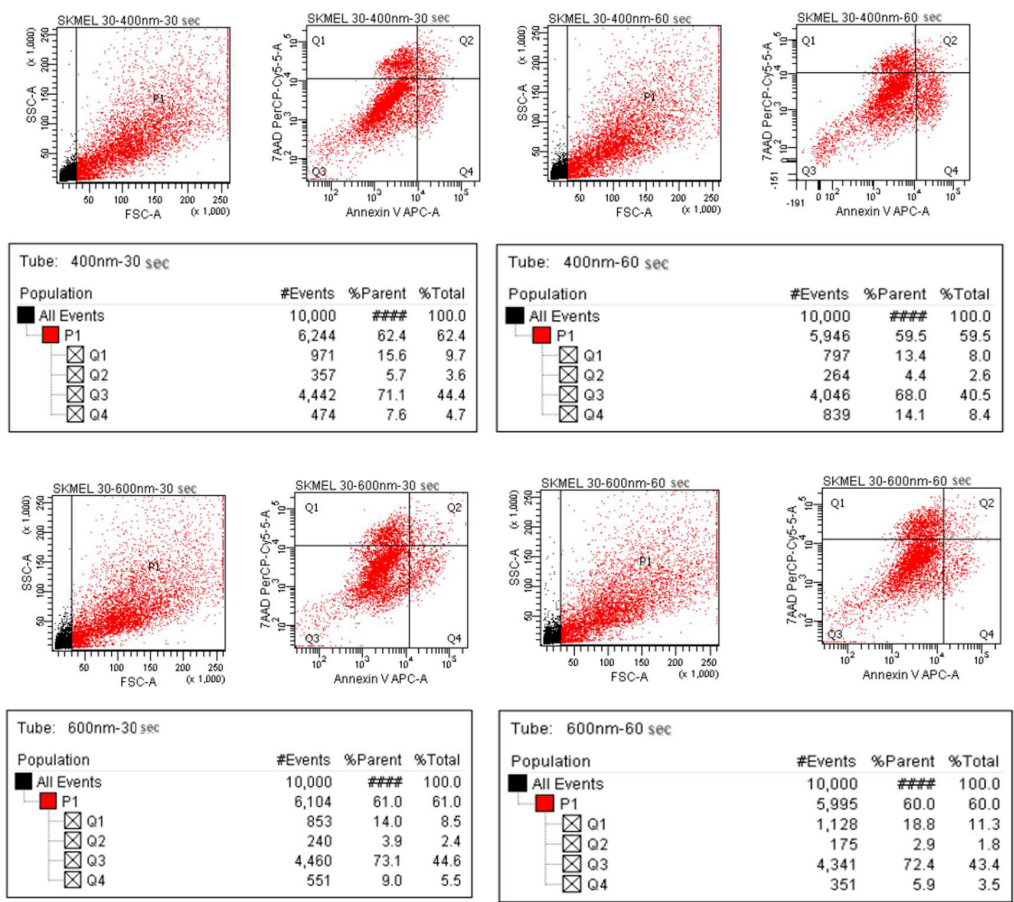

Figure 4. Annexin-V/7-AAD apoptosis/necrosis analysis of $30 \mathrm{~s}$ and $60 \mathrm{~s}$ PDT treated SK-MEL-30 cells.
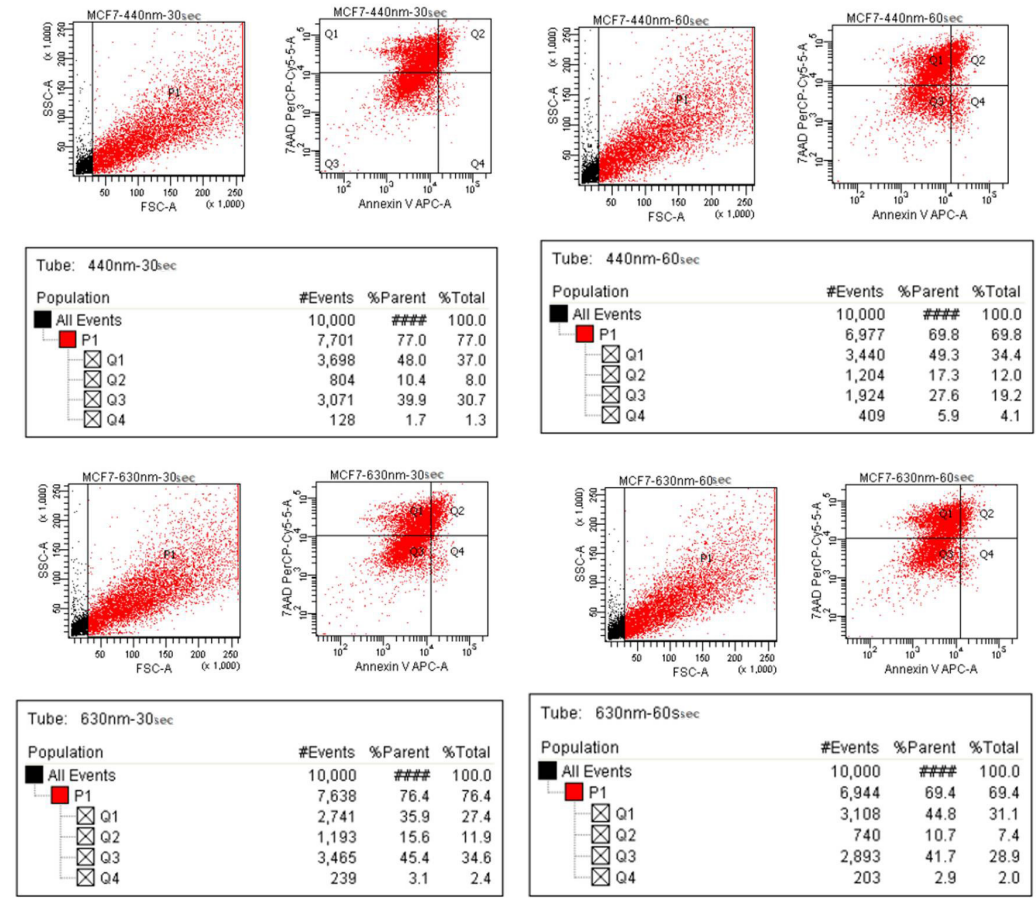

Figure 5. Annexin-V/7-AAD apoptosis/necrosis analysis of $30 \mathrm{~s}$ and $60 \mathrm{~s}$ PDT treated MCF-7 cells. 
Table 1. Representation of \% cell populations that are living and that experience cell death through apoptosis and necrosis after PDT. ( ${ }^{*}$ Considerable cell death percentages)

\begin{tabular}{|c|c|c|c|c|c|c|c|c|c|c|c|c|c|}
\hline & & \multicolumn{12}{|c|}{ \% Cell population (Flow cytometry analysis) } \\
\hline & & \multicolumn{2}{|c|}{ UMUC-3 } & \multicolumn{2}{|c|}{ SKMEL-30 } & \multicolumn{2}{|c|}{ MCF-7 } & \multicolumn{2}{|c|}{ UMUC-3 } & \multicolumn{2}{|c|}{ SKMEL-30 } & \multicolumn{2}{|c|}{ MCF-7 } \\
\hline \multirow{2}{*}{\multicolumn{2}{|c|}{$\begin{array}{l}\text { Fs laser wavelength } \\
\text { Irradiation duration }\end{array}$}} & \multicolumn{2}{|c|}{$420 \mathrm{~nm}$} & \multicolumn{2}{|c|}{$630 \mathrm{~nm}$} & \multicolumn{2}{|c|}{$400 \mathrm{~nm}$} & \multicolumn{2}{|c|}{$600 \mathrm{~nm}$} & \multicolumn{2}{|c|}{$440 \mathrm{~nm}$} & \multicolumn{2}{|c|}{$630 \mathrm{~nm}$} \\
\hline & & $30 \mathrm{~s}$ & $60 s$ & 30 s & $60 s$ & $30 s$ & $60 s$ & 30 s & $60 s$ & 30 s & $60 s$ & 30 s & $60 s$ \\
\hline Q3 & Living & 26.4 & 31.5 & 60.9 & 59.2 & 71.1 & 68.0 & 73.1 & 72.4 & 39.9 & 27.6 & 45.4 & 41.7 \\
\hline Q4 & Apoptosis & 41.1 & 37.9 & 21.1 & 22.5 & 7.6 & 14.1 & 9.0 & 5.9 & 1.7 & 5.9 & 3.1 & 2.90 \\
\hline $\begin{array}{l}\text { Q1 } \\
\text { Q2 }\end{array}$ & $\begin{array}{c}\text { Late Apoptosis \& } \\
\text { Necrosis }\end{array}$ & 32.4 & 30.6 & 18.0 & 18.3 & 21.3 & 17.8 & 17.9 & 21.7 & 58.4 & 66.6 & 51.5 & 55.5 \\
\hline & otal Cell Death & $\star 73.5$ & *68.5 & 39.1 & 40.8 & 28.9 & 31.9 & 26.9 & 27.6 & *60.1 & $* 72.5$ & 54.2 & 58.4 \\
\hline
\end{tabular}
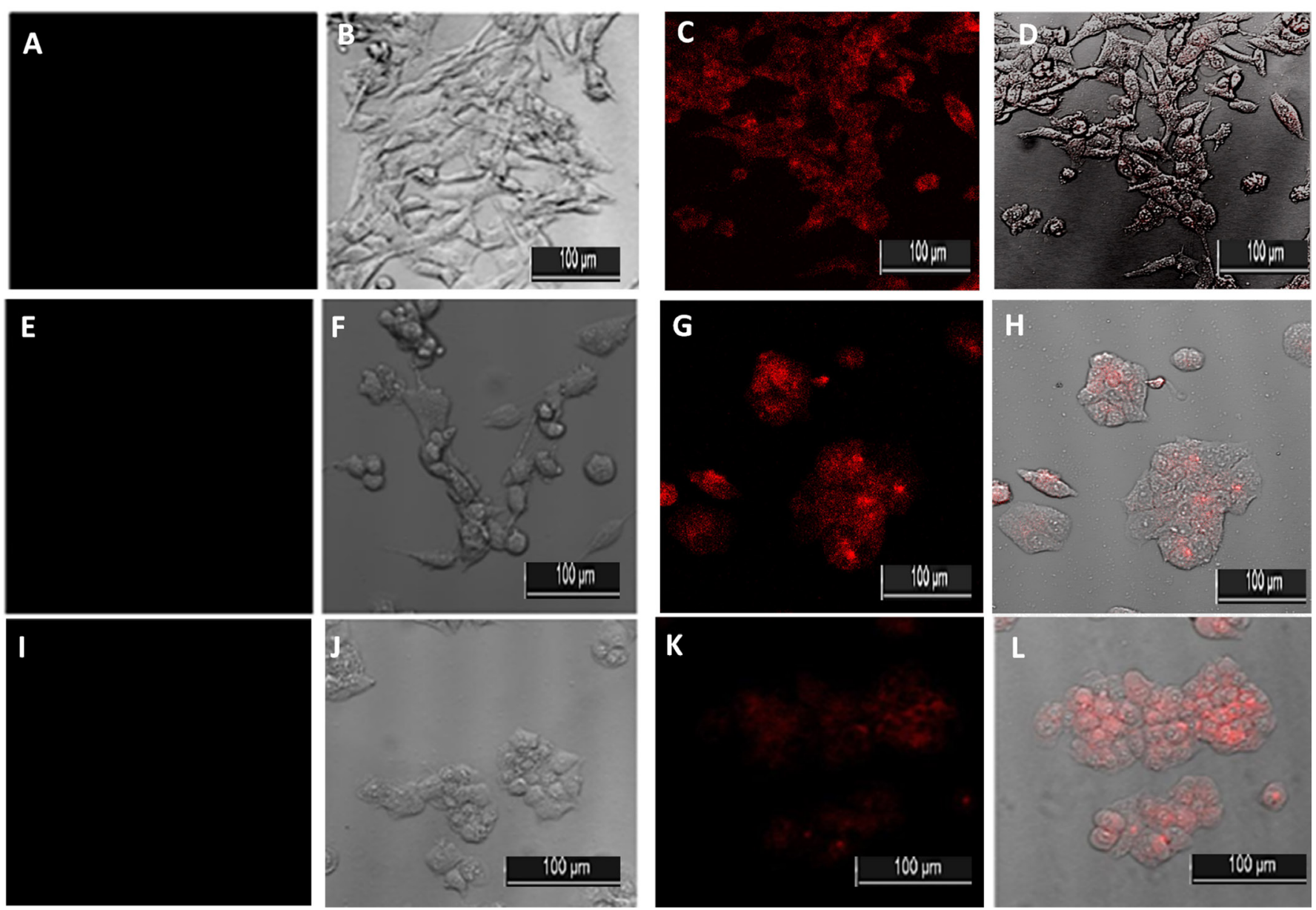

Figure 6. Intracellular fluorescent (PpIX) accumulation induced by ALA treatment observed after PBS washes, (40X-oil, 512x512). A) UMUC-3 cells non-ALA treated, observed by confocal microscope with standard detector-SD; B) UMUC-3 cells non-ALA treated, observed in white field by transmitted detector-TD; C) ALA treated UMUC-3 cells, observed by confocal microscope with SD; D) ALA treated UMUC-3 cells observed by white field and confocal with SD and TD; E) SK-MEL-30 cells non-ALA treated, observed by confocal microscope with SD; F) SK-MEL-30 cells non-ALA treated, observed in white field by TD; G) ALA treated SK-MEL-30 cells, observed by confocal microscope with SD; H) ALA treated SK-MEL-30 cells observed by white field and confocal with SD and TD; I) MCF-7 cells non-ALA treated, observed by confocal microscope with SD; J) MCF-7 cells non-ALA treated, observed in white field by TD; K) ALA treated MCF-7 cells, observed by confocal microscope with SD; L) ALA treated MCF-7 cells, observed by white field and confocal with SD and TD. (Excitation: $405 \mathrm{~nm}$; emission: $614 \mathrm{~nm}$ ) 
vide the low light toxicity for the normal surrounding cells and also short therapy periods. It may be proposed that, the 5-ALA mediated, fs laser PDT may be targeted therapy with early response for bladder cancer and breast cancer. On the other side, these suggestions need to be proven by in-vivo tests.

Cell death after ALA-PDT with continuous light for 125 and 500 seconds was proved to be occurred due to apoptosis originated from alterations in JAK/STAT pathway and Bcl-2, Bax gene expression levels in A431 and COLO-6 cells (24). Di Venosa et al. previously claimed that THP-ALA has a good systemic distribution to be used for brain tumors and they have also proved that ALA derivatives have high topical infusion rate and stability to be used for skin cancers (25). It is known that, activation of photosensitizer by any light source; continuous or pulsed; the reactive oxygen radicals develop in the presence of oxygen. These species oxidize cellular components, causing apoptosis and necrosis (26). It means that here the key factor is activating the photosensitizing molecule by any light source. On the other side, it would be preferred that duration of irradiation would be short, energy of the light is effective to cause cancer cell death and the conditions are safe for neighboring healthy cells. In this study we regarded the PDT conditions that allow cell viability at most $50-60 \%$ and at which the control cell viability is high as "the efficient condition", additionally other parameters on real tissue experiments should be performed and optimization of the parameters for photo-chemotherapy may be considered in that more narrow range in future studies. Here the conditions that the cells from different cancer sources experience cell death through apoptosis and/or necrosis after the fs-PDT were reported. On the other hand, the molecular mechanisms of the PDT resistance, the responsible proteins and the genes should be investigated in detail in coming studies.

It was reported that, altered activities of heme biosynthesis enzymes, functions of mitochondria elements and activities of membrane transporters may be different in different cancer types (27). These parameters contribute to ALA mediated protoporphyrin accumulation in tumor cells. ALA mediated cancer diagnosis may be enhanced by molecular modulations. So, here our results revealed that ALA mediated PpIX accumulation may be used in diagnostic applications in skin, bladder and breast cancer in the experimental parameters used in our work. Barcessat et al. tested ALA mediated PDT with diode laser at a wavelength $660 \mathrm{~nm}, 40 \mathrm{~mW}$ power, $90 \mathrm{~J} / \mathrm{cm}^{2}$ fluency for $1.5 \mathrm{~min}$ in each point to treat oral lesions (28). They found that two sessions of ALA mediated PDT with indicated induction parameters was not enough for total remission of the lesion. They claimed that more PDT sessions must be applied to guarantee the total healing. The light source is important parameter in PDT; it is known that non-coherent light sources are easy to use and relatively cheap. However, lasers are useful as they produce mono-chromatic light of a predetermined wavelength, light dose can easily be calculated and the light can reach through the optical fiber to the target location (16). Hammer et al. claimed that femtosecond pulses are comparatively safe, having high threshold for laser-induced breakdown in aqueous matrix (29). Actually their results demonstrate that the predominating breakdown process of the fs pulse is multi-photon breakdown of water and this is not dependent to the existence of electrolytes. According to literature, pulsed light offers potential advantages since pulsed lasers can generate low heating in the target tissue and neighboring tissue. Sometimes delivering light to deeper tissue may be preferred, like in cases of breast cancer tumors. In such cases increased power is needed to develop required energy at tumor site and that much power may result in tissue at the surface layer of the target tissue. In such circumstances pulsed light may be more effective (30). Hashmi et al. claimed that the "quench period" when the laser pulse is off, reduces heating and allows the use of much higher power intensity that may be safely applied to tissue without pain. In this study, we used femtosecond laser as a light source. To sum up, fs-laser has potential to be used as a light source for cancer therapy by PDT in clinic.

\section{Final remarks}

According to our findings, all the cancer cells were not cleared with $100 \%$ cell death. By selecting the possible effective wavelengths, we developed a background for the future studies. Accordingly, the parameters may be altered. Also it would be enlightening to focus on the gene expression analysis at the most effective parameters. The $400 \mathrm{~nm}$ and $630 \mathrm{~nm}$ ALA mediated fs-PDT should be deeply studied to exhibit the other cellular and molecular analysis. Collectively, $70 \%$ cell death was reached that may be assumed as a good beginning for a therapeutic strategy for the cancer types we worked in this paper. At this point another question will be if the melanoma cells have exhibited resistance to ALA mediated fs-PDT. This is the first report which evaluates the ALA mediated PDT in different cancer cells with different fs laser wavelengths as light source. The results demonstrate that ALA and femtosecond laser mediated PDT may be used together as therapeutic and diagnostic tool to target different cancer types. Here, it is confirmed that the wavelength of laser treatment and treatment duration are important parameters that determine the destiny of ALA mediated fs laser-photodynamic therapy.

\section{Disclosure statement}

The authors declare that there is no conflict of interest.

\section{Funding}

This study was funded by The Scientific and Technological Research Council of Turkey (TUBITAK project no_115Z002).

\section{Acknowledgement}

Selçuk University Research Centers ILTEK \& SULTAN are gratefully acknowledged.

\section{References}

1. Korte F, Nolte S, Chichkov BN, Bauer T, Kamlage G, Waner T, Fallnich C, Welling H. Far-field and near-field mate- 
rial processing with femtosecond laser pulses. Appl Phys A 1999; 69: doi:10.1007/s003399900391.

2. Serbin J, Bauer T, Fallnich C, Kasenbacher A, Arnold WH. Femtosecond lasers as novel tool in dental surgery. Applied Surface Sci 2002; 197: 737-40.

3. Atif M, Dyer PE, Paget TA, Snelling HV, Stringer MR.Two-photon excitation studies of $\mathrm{m}$-THPC photosensitizer and photodynamic activity in an epithelial cell line. Photodiagnosis Photodyn Ther 2007; 4: 106-11.

4. Tandan S, Lamba AK, Faraz F, Aggarwal K, Chowdhri K. A case report of bisphosphonate related osteonecrosis of the jaw treated byphotodynamic therapy. Photodiagnosis Photodyn Ther 2019; 26: 313-5.

5. American Cancer Society web page. https://www.cancer.org/treatment/ treatments -and-side-effects/treatment-types/photodynamic-therapy.html, [cited 2020 May 03].

6. Singer A, Markoutsa E, Limayem A, Mohapatra SS. Nanobiotechnology medical applications: Overcoming challenges through innovation. The EuroBiotech J 2018; 2: 146-60.

7. Ferlay J, Colombet M, Soerjomataram I, Dyba T, Randi G, Bettio M, Gavin A, Visser O, Bray F. Cancer incidence and mortality patterns in Europe: Estimates for 40 countries and 25 major cancers in 2018. Eur J Cancer. 2018; 103: 356-87.

8. Kawczyk-Krupka A, Bugaj AM, Latos W, Zaremba K, Wawrzyniec K, Kucharzewski M, Sieron' A. Photodynamic therapy in colorectal cancer treatment-The state of the art in preclinical research. Photodiagnosis Photodyn Ther 2015; doi:10.1016/j.pdpdt.2015.07.175.

9. Bugaj AM. Targeted photodynamic therapy—a promising modality of cancer treatment. Photochem Photobiol Sci 2011; 10: 1097-109.

10. Kiesslich T, Krammer B, Plaetzer K. Cellular mechanisms and prospective applications of hypericin in photodynamic therapy. Curr Med Chem 2006; 13: 2189-204.

11. Kars MD, Kara R, Gündoğdu Y, Kepceoğlu A, Kılıç HŞ. Femtosecond laser induced photodynamic therapy on 5-ALA treated SKMEL-30 cells: an efficient theranostic strategy to combat melanoma. Biomed Pharmacother. 2014, 68: 657-62.

12. Kars G, Ceylan A. Biohydrogen and 5-aminolevulinic acid production from waste barley by Rhodobacter sphaeroides O.U.001 in a biorefinery concept. Int J Hydrogen Energ 2013; 38: 5573-9.

13. Iinuma S, Farshi SS, Ortel B, Hasan T. A Mechanistic Study of Cellular Photodestruction with 5-Aminolevulinic Acid-Induced Porphyrin. Br J Cancer 1994; 70: 21-8.

14. Lang K, Bolsen K, Stahl W, Ruzicka T, Sies H, Lehmann P, Fritsch C. The 5-aminolevulinic acid-induced porphyrin biosynthesis in benign and malignant cells of the skin. J Photochem Photobiol B 2001; 65: 29-34.

15. Li Y, Wang P, Zhao P, Zhu S, Wang X, Liu Q. Apoptosis induced by sonodynamic treatment by protoporphyrin IX on MDA-MB-231 cells. Ultrasonics 2012; 52: 490-6.

16. Taylor EL, Brown SB. The advantages of aminolevulinic acid photodynamic therapy in dermatology. J Dermatolog Treat 2002; 13: 3-11.
17. Ackroyd R, Kelty C, Brown N, Reed M. The History of Photodetection and Photodynamic Therapy. Photochem Photobiol 2001; 74: 656-69.

18. Kars G, Alparslan Ü. Valorization of sugar beet molasses for the production of biohydrogen and 5-aminolevulinic acid by Rhodobacter sphaeroides O.U.001 in a biorefinery concept. Int J Hydrogen Energ 2013; 38: 14488-94.

19. Krammer $B$, Uberriegler $K$. In-vitro investigation of ALA-induced protoporphyrin IX. J Photochem Photobiol B 1996; 36: 121-6.

20. Schmitz L, Novak B, Hoeh AK, Luebbert H, Dirschka T. Epidermal penetration and protoporphyrin IX formation of two different 5-aminolevulinic acid formulations in ex vivo human skin. Photodiagnosis Photodyn Ther 2016; 14: 40-6.

21. Palasuberniam $\mathrm{P}$, Yang $\mathrm{X}$, Kraus D, Jones $\mathrm{P}$, Myers KA, Chen B. ABCG2 transporter inhibitor restores the sensitivity of triple negative breast cancer cells to aminolevulinic acid-mediated photodynamic therapy. Sci Rep 2015; doi:10.1038/srep13298.

22. Murtagh J. Femtosecond Laser-Assisted Cataract Surgery (FLACS): Issues in emerging health technologies. Ottawa: Canadian Agency for Drugs and Technologies in Health. 2014; pp 126.

23. Bogoeva V, Siksjø M, Saeterbø KG, Melo TB, Bjorkoy A, Lindgren M, Gederaas OA. Ruthenium porphyrin-induced photodamage in bladder cancer cells. Photodiagnosis Photodyn Ther 2016; doi: 10.1016/j.pdpdt.2016.01.012.

24. Qiao L, Mei Z, Yang Z, Li X, Cai H, Liu W. ALA-PDT inhibits proliferation and promotes apoptosis of SCC cells through STAT3 Signal Pathway. Photodiagnosis Photodyn Ther 2016; doi: 10.1016/j.pdpdt.2016.01.008.

25. Di Venosa G, Batlle A, Fukuda H, Macrobert A, Casas A. Distribution of 5-aminolevulinic acid derivatives and induced porphyrin kinetics in mice tissues. Cancer Chemother Pharmacol 2006; 58: 478-86.

26. Wan MT, Lin JY. Current evidence and applications of photodynamic therapy in dermatology. Clin Cosmet Investig Dermatol. 2014; 21, 7: 145-63.

27. Yang X, Palasuberniam P, Kraus D, Chen B. Aminolevulinic Acid-Based Tumor Detection and Therapy: Molecular Mechanisms and Strategies for Enhancement. Int J Mol Sci. 2015; 16: 25865-80.

28. Barcessat AR, Huang I, Rosin FP, dos Santos Pinto D Jr, Maria Zezell D, Correa L. Effect of topical 5-ALA mediated photodynamic therapy on proliferation index of keratinocytes in 4-NQO-induced potentially malignant oral lesions. J Photochem Photobiol B 2013; 126: 33-41.

29. Hammer DX, Thomas R J, Noojin GD, Rockwell BA, Kennedy PK, Roach WP. Experimental investigation of ultrashort pulse laser-induced breakdown thresholds in aqueous media. IEEE J Quant Electron 1996; 32: 670-8.

30. Hashmi JT, Huang YY, Sharma SK, Kurup DB, De Taboada L, Carroll JD, Hamblin MR. Effect of pulsing in low-level light therapy. Lasers Surg Med 2010; 42: 450-66. 